\title{
Philosophiques
}

\section{Pour une logique de l'action tragique : Hegel et la tragédie}

\section{Pierre Gravel}

Volume 5, numéro 1, avril 1978

URI : https://id.erudit.org/iderudit/203086ar

DOI : https://doi.org/10.7202/203086ar

Aller au sommaire du numéro

Éditeur(s)

Société de philosophie du Québec

ISSN

0316-2923 (imprimé)

1492-1391 (numérique)

Découvrir la revue

Citer cet article

Gravel, P. (1978). Pour une logique de l'action tragique : Hegel et la tragédie.

Philosophiques, 5(1), 111-131. https://doi.org/10.7202/203086ar d'utilisation que vous pouvez consulter en ligne.

https://apropos.erudit.org/fr/usagers/politique-dutilisation/ 


\title{
POUR UNE LOGIQUE DE L'ACTION TRAGIQUE : HEGEL ET LA TRAGEDIE

\author{
par Pierre Gravel
}

\begin{abstract}
«Nous avons ici dans l'art un mode de manifestation particulier de l'esprit. L'art est une forme particulière sous laquelle l'esprit se manifeste, car il peut pour se manifester revêtir d'autres formes encore, etc. " ${ }^{1}$
\end{abstract}

La Tragédie occupe, dans l'œuvre de Hegel, une place particulière et particulièrement importante ; en témoigne la régularité quasi obsédante de son retour ${ }^{2}$. Et pourtant, force est de conclure que cette particularité, - voire dans le creux de certains passages cette singularité de la Tragédie, - n'est pas inquiétante. Voilà qui est suffisamment étonnant pour insister : pourquoi cette répétition? Pourquoi cette insistance à revenir sur ce qui n'est, après tout, que l'un des modes possibles de la manifestation du soi, alors que, par exemple et de manière tout à fait générale, "dans la hiérarchie des moyens servant à exprimer l'absolu, la religion et la culture issue de la raison occupent le degré le plus élevé, bien supérieur à celui de l'art " ?3 Moment particulier et particulièrement significatif du devenir soi $d u$ soi dans cette grande fresque des stations principales de l'esprit qu'est la Phénoménologie, mode absolument formidable de la manifestation de l'esprit dans les textes de l'Esthétique, la Tragédie pourtant est non seulement destinée à disparaître, c'est à partir du point anticipé de sa disparition qu'elle est rappelée. L'étrangeté vaut ici d'être soulignée : à supposer que

1. Hegel, Introduction à l'Estbétique, Paris, Aubier, 1964, p. 19.

2. Les principaux textes ont été colligés en anglais dans un excellent ouvrage intitulé : Hegel on Tragedy, New York, Harper and Row, 1975, 404 pages. Comment comprendre pourtant l'étonnant oubli de l'article de 1801 sur le Droit Naturel? Il s'agit là de l'un des premiers textes sur la Tragédie où l'ensemble de la doctrine est conquis. A ce titre seul, les pages 130 à 134 de l'édition française méritent un traitement particulier, ce qu'a très bien vu Szondi dans son ouvrage Poésie et Poétique de l'idéalisme allemand, Paris 1974, p. 9 sq.

3. Hegel, Introduction à l'Esthétique, ibid, p. 42. 
l'art puisse encore nous être question, pourquoi cette insistance à répéter ce mode particulier de manifestation à partir de l'anticipation, et nous ajouterions : de l'anticipation attisée de sa disparition? Car étrangement, s'il s'agit, pour Hegel, d'évoquer à nouveau le jeu de la puissance à l'œuvre dans la Tragédie, ce n'est pas pour jouer du pur pouvoir de l'évocation, ce n'est pas, non plus, rappeler les vertus et les charmes du travail de l'anamnèse, ce n' est pas, enfin, répéter la sourde puissance d'un Oublié fondamental dont les traits principaux, d'être rappelés, pourraient toujours nous concerner ; il faut plutôt entendre que cette nouvelle évocation, cet à nouveau de l'évocation, cette ré-évocation, si elle n'est d'emblée révocation, a du moins lieu, source et sens à partir de et dans l'anticipation de sa révocation. Cette situation de la Tragédie dans le rapport au devenir soi du soi, pour reprendre les termes de la Phénoménologie, cette étrange situation où le sens du tragique est lu à partir du non-tragique de sa disparition ou de sa relève (Aufbebung), nous la considérons comme étant éminemment exemplaire ou symbolique du statut général qui, dans le même discours, est attribué à l'art : préfiguration de ce qui de soi n'est pas depuis le besoin de la figure, ou, ce qui revient au même, de ce qui peut être depuis le besoin de la figure tant et aussi longtemps qu'il ne s'est pas compris selon la nécessité de son être essentiel : l'être auprès de soi dans le surmontement ou la sursomption accomplie (Aufbebung) de toutes les contradictions. Car, pour Hegel comme pour la pensée juive, l'Alliance, c'est-à-dire la promesse infinie et infiniment reportée de l'infinité du rapport de soi à soi, n'admet aucun arrêt ou aucune stase en une figuration privilégiée. Le terme est l'au-delà de toute limite et c'est dans l'en-vue de son franchissement déjà su que toute limite est pratiquée. Ce qui nous semble ici tout à fait remarquable, et ce sur quoi il faut insister, c'est que le discours sur l'esthétique, au moment même où il atteint avec Hegel la forme achevée qui est la sienne, est sinon possible sur le fondement, du moins contemporain de la déclaration et de la constatation de la mort, c'est-à-dire de l'in-intérêt fondamental, de son objet. L'in-intérêt fondamental, ici, cela veut simplement dire que l'art, et même dans ses manifestations les plus vives, ne saurait atteindre ce que Hegel appelle l'" Esprit "dans ses fondements, en d'autres termes, ne saurait plus être source d'un retournement'.

4. Phénoménologie, p. 71.

5. Comme ce sera par exemple le cas chez Hölderlin, et plus près de nous chez Nietzsche et chez Heidegger. 
Car il faut, croyons-nous, relire et méditer avec soin cette déclaration qui termine le premier chapitre d'ouverture de l'Introduction à l'Esthétique :

"Sous tous ces rapports, l'art reste pour nous, quant à sa suprême destination, une chose du passé. De ce fait, il a perdu pour nous tout ce qu'il avait d'authentiquement vrai et vivant, sa réalité et sa nécessité de jadis, et se trouve désormais relégué dans notre représentation. Ce qu'une ceuvre d'art suscite aujourd'hui en nous, c'est, en même temps qu'une jouissance directe, un jugement portant aussi bien sur le contenu que sur les moyens d'expression et sur le degré d'adéquation de l'expression au contenu $"{ }^{6}$

On peut donner de ce passage de nombreuses interprétations et, à en étendre la signification, lui conférer des sens différents, voire même contradictoires; nous aimerions simplement souligner ceci - et uniquement pour en prendre la mesure - qu'à la lettre le discours sur l'esthétique est possible sur le fondement de l'inexistence fondamentale de son objet. Il y a là une situation tout à fait particulière et qui est celle de l'esthétique. Si on a pu dire, en effet, qu'avec l'ethnologie on avait le prototype, en quelque sorte, d'un moď̀le de rationalité qui avait activement travaillé à la destruction effective de son "objet" réel, et donc de " cela " qui l'avait tout d'abord suscité à se constituer en projet de savoir et pouvait continuer d'en justifier la nécessité : les sociétés dites " primitives $"{ }^{7}$, avec l'esthétique, cette fois, on a quelque chose de tout à fait différent et peut-être même de plus fondamentalement difficile : un type de rationalité dont le moment même de la constitution est celui de la mise à l'écart de son objet, c'est-à-dire et pour une bonne part de ses propres modes d'appréhension et de saisie de ce qui est censé être son objet ${ }^{8}$. Quoi qu'il en soit, ce que nous aimerions tenter, c'est, à l'occasion du discours hégélien et sur le cas précis de l'étude de la Tragédie, d'ouvrir et d'occuper quelque peu l'espace qui sépare et disjoint la répétition de l'évocation de la déclaration de la révocation; c'est tenter de rendre manifestes quelques-uns des liens qui rendent possible la constitution de l'un

6. Hegel, Introduction à l'Estbétique, t.c. p. 43.

7. On peut renvoyer, ici, aux remarquables travaux de P. Clastres (v.g. La Société contre l'État, Paris, Minuit) ; à ceux également de Lefort et de Castoriadis, et plus particulièrement à ce premier numéro de cette revue très récente (mars 1977) intitulée Libre (Payot).

8. Nous pensons ici tout particulièrement au modèle platonicien comme première figuration achevée du désir de savoir qui est contemporain, en son institution, de l'exclusion de la poésie. Plus précisément, c'est sur et à partir de cette exclusion qu'il entend se donner son plus grand titre de gloire. Cf. République $X$. 
dans l'oubli, sur le fondement, ou dans l'anticipation de l'autre. Peut-être se peut-il, en effet, que la situation générale de l'art dans le rapport à ce qu'on appelle la pensée soit de l'ordre de ce que Hegel nous apprend à penser - Hegel serait ainsi une figure exemplaire de ce qui est encore un incontournable de notre savoir - ; cela toutefois devrait nous inviter à continuer de la questionner, et, comme pour le forgeron de la fable, à cent fois sur le métier remettre l'ouvrage. Et soupçonner qu'il y a là suffisamment sinon pour la position, du moins pour la frappe, d'une question. On peut en effet lire les termes du désir de justice qui semble animer l'bomo rationalis aux maximes qu'il inscrit au fronton de ses temples et sur les pierres polies de ses palais dits de "justice" on peut également les lire aux signes qu'il trace sur les corps de ses suppliciés, aux opprobres dont il charge ceux qu'il désire exclure, ou encore à la manière toute particulière suivant laquelle il s'occupe de ceux qu'il daigne considérer. Dans le premier cas, on y lit ce qu'il pose à l'infini de lui-même comme le terme accompli de son désir ; dans le second, ce qui doit être nécessairement fait, puisqu'il l'est de toute façon, pour le pur maintien de ce désir. On voit dès lors notre question: quand la machine de lecture hégélienne prend en charge la Tragédie, comment s'occupe-t-elle alors de ce qui est censé la concerner? Comment et au nom de quoi s'acquittet-elle alors de ce dont elle s'occupe? D'un parcours de ce problème, nous isolerons les points principaux pour en mettre enfin les termes en question.

De l'ensemble des textes de Hegel qui portent sur la Tragédie, deux, principalement, seront étudiés. La raison en est simple : à la différence de ces nombreux textes, par exemple, de la philosophie du droit ou de la philosophie de l'histoire, où la Tragédie est étudiée comme illustration d'un point de la doctrine, il existe deux textes principaux où la Tragédie est non seulement intégrée au mouvement et au développement de l'ensemble, mais où elle est partie prenante et moment essentiel de ce procès - et de ce procès gagné - qui a nom philosophie. Il s'agit, on l'aura deviné, de l'Esthétique, plus particulièrement du huitième volume de l'édition française qui est consacré au développement de la poésie, et de la Phénoménologie, plus particulièrement de son avant-dernier chapitre, ce par quoi nous allons commencer.

Dans la Phénoménologie, en effet, à part quelques appari- 
tions sporadiques, l'étude de la Tragédie nous est donnée principalement comme partie de l'examen de la religion, plus précisément au chapitre de la religion esthétique, entre-deux de la religion dite " naturelle " et de la religion révélée, celle-ci étant révélation de l'esprit de la première, la religion elle-même étant la conscience de soi de l'esprit, ou, si l'on préfère, le lieu véritable du devenir soi du soi. Le fond est connu, mais peut-être convient-il d'abord de le rappeler : le ressort de la dialectique qui sera mise en œuvre à propos de la Tragédie non seulement en provient et s'en détache, mais encore devra y être ramené pour enfin disparaître.

La conscience - entendre, pour être plus près de l'Allemand : l'être conscient, ou le fait d'être conscient (Bewusst-Sein), cette doublure du réel dans le réel à partir d'où la réalité même du réel est en question et trouve à se décider - la conscience, donc, tout au long de la Phénoménologie, fait de soi et sur soi une expérience essentiellement négative. Tout se passe, en effet, comme si elle était pour elle-même la matrice de l'épreuve de son propre devenir. Cette épreuve qui, tant qu'elle n'est pas comprise, c'est-àdire portée et assumée jusqu'à son terme, est celle de la douleur, du désespoir et du scepticisme 9 . La formule de cette expérience, qui, pour Hegel, est celle de la pure négativité, c'est-à-dire de la perte éprouvée de l'essentiel ${ }^{10}$, est la suivante : la conscience est depuis un autre de l'antécédence et de la reconnaissance duquel elle a besoin pour être pleinement selon les termes de ce savoir non encore véritablement su qu'elle a pourtant déjà d'elle-même ; dans l'expérience de détermination et de saisie qu'elle tente de faire de cet autre, elle découvre, à son corps défendant, qu'elle est, et elle seule, de cet autre la détermination fondamentale ; en d'autres termes, la conscience découvre que c'est non seulement pour elle qu'il y a un autre, mais encore qu'en étant elle-même le même pouvoir de la détermination de cet autre en l'élément même de son altérité, cela fait qu'il n'y a tout simplement pas de véritable autre. Cette expérience de pure négativité, de pure perte et de pure dépossession, où le nom le plus propre du réel est peut-être celui, double, de la fuite et de la répétition, se transforme alors et affecte la conscience en son centre : du pur vouloir de la détermination et

\footnotetext{
9. Cf. principalement Phénoménologie, p. 69. Cf. également de ces passages au ton très kierkégaardien le commentaire que donne Heidegger in Hegel et son Concept de l'Expérience (Chemins qui ne mènent nulle part, Paris, Gallimard, 1962, pp. 101 à 172).

10. Phénoménologie, p. 69 sq.
} 
de la saisie de l'autre, la conscience s'éprouve comme désir ou comme puissance désirante, c'est-à-dire comme manque de l'autre duquel elle attendait la grâce et la sanction de la reconnaissance. De l'autre muet dans l'élément de la pure extériorité dont le moment de la détermination n'est finalement, pour la conscience, que celui de sa propre répétition, la conscience attend désormais un autre loquace sous la figure cette fois d'autrui ; elle se donne alors cet autre comme un autre elle-même et engage aussitôt avec lui la lutte pour la reconnaissance; nouvelle épreuve au terme de laquelle elle apprendra que le don de l'extériorité et la grâce de l'altérité qui confirment, elle se les était donnés à elle-même, mais ne savait pas le voir, par ce rapport à la négativité qui s'appelle travail ou formation (Bildung), grâce au creuset duquel ce qui peut advenir se donne. Le passage à l'esprit de la religion tient à ceci que l'autre en l'élément de son altérité dont le manque avait été éprouvé dans tous les stades antérieurs, ce manque dont l'expérience avait été pour la conscience l'occasion de son inscription dans le registre du désir, puis, par après, celle de son élévation au niveau de la moralité, cet autre doit d'abord devenir puissance substantielle comme figure solide et autonome de la totalité en soi, c'est-à-dire l'infinité de l'élément divin lui-même, puis, dans un deuxième moment, il faut que cette réalité substantielle reçoive de surcroît le pouvoir et la puissance du Soi. En somme, pour que l'esprit de la religion s'accomplisse véritablement, il faut que là aussi la substance devienne sujet. La Tragédie, dans l'étude qu'en donne la Phénoménologie, est l'intervalle précis dans, par et à travers lequel s'accomplit la décomposition de la réalité substantielle du divin, cette décomposition qui est également, mais comme son envers, son élévation à la pure position d'un sujet conscient de soi comme de la totalité de ce qui est. Ce passage n'est pas aussi innocent qu'il le pourrait paraître : s'y perd, en effet, la nécessité de la figuration - le sujet, à la différence de la substance, n'est pas représentable - ; s'y gagne, par contre, ${ }^{1}$ la substitution à l'esprit du paganisme de ce qui est considéré comme l'essentiel de l'esprit chrétien. En d'autres termes, dans et par la Tragédie, se transforme le sens du divin: aux sacrifices d'Oedipe, d'Antigone et d'Iphigénie qui sont révélation, sur le dos de l'homme, de la réalité substantielle du divin comme réalité de la loi morale, se substitue

11. Cf. de ce "gain " le commentaire de G. Lebrun in Hegel, La Patience du Concept, Paris, Gallimard 1972, p. 101 sq. 
le sacrifice du Christ, qui est manifestation, accomplie sur le dos du dieu lui-même, de l'infinité traversée et renversée du sujet substantiel qui est véritablement capable de sa transfiguration. La Tragédie est ainsi lieu matriciel, comme et en tant qu'occasion d'un chiasme : au mouvement de chute et de descendance par quoi s'accomplit la décomposition de la substantialité divine, - le dieu présent dans les éléments: les arbres, la force de la nature, le soleil, les pierres, etc., - correspond son élévation et sa transfiguration dans l'accession et l'ascension à la conscience de soi. Car tout se passe, en effet, comme si la Tragédie avait pour fin et fonction principales d'accomplir la suppression de l'esprit divin dans l'élément corporel de la naturalité par la naissance, la production et l'accession de la conscience de soi, conscience de soi qui devra à son tour gagner sa suppression dans l'élément de la religion révélée.

Dans l'Esthétique, la Tragédie est analysée de façon analogue, à la différence toutefois qu'au lieu d'être présentée comme le second moment d'un procès dialectique, celui de l'antithèse, qui doit gagner sa suppression dans l'élément de résomption qu'est la synthèse, depuis l'antécédence duquel il peut et même doit être lu - ainsi, pour le Hegel de la Phénoménologie, il ne fait aucun doute qu'Antigone est déjà une préfiguration du Christianisme-, dans l'Estbétique, donc, la Tragédie apparaît plutôt comme le troisième moment du procès dialectique, c'est-à-dire comme moment de la synthèse de deux moments analysés antérieurement: le stade épique et le stade lyrique, c'est-à-dire essentiellement Homère et Pindare. D'un côté : description objective de l'individualité qui est littéralement "prise " dans la réalité où chaque particularité est révélation de l'essentiel dans sa substantialité ; de l'autre: manifestation de l'intériorité de quelque chose comme un moi qui n'aurait pas encore trouvé dans le réel son pendant et son répondant. La Tragédie, comme troisième moment, opère la synthèse du lyrique et du descriptif. Cette différence vaut en effet d'être notée: se gagne, dans un des cas, celui de la Phénoménologie, une suppression au nom d'un autre, le devenir soi du soi réalisé dans le christianisme; se gagne dans l'autre cas, celui de l'Esthétique, une suppression finale. Le mouvement téléologique qui commande les deux analyses n'est donc pas tout à fait le même : dans le premier cas, le télos l'est d'un autre qu'il anticipe et préfigure, et d'un autre qui en est tout aussi bien la règle, puisque 
c'est pour et vers l'accession de cet autre que l'analyse est menée et que la suppression se gagne; alors que dans le second cas, le télos est aussi un "eschaton", pour reprendre la distinction aristotélicienne $^{12}$, et, comme tel, ne peut donner lieu ou naissance à aucune relève. Ainsi, le penseur qui, dans l'Esthétique, annonce et pense la fin de l'art, est peut-être également celui à partir duquel on peut penser l'art comme fin ${ }^{13}$. Toutefois, cette différence dans la présentation, cette différence dans le fond depuis lequel la perspective se découpe, n'entame véritablement ni la forme, ni la contenu de l'analyse qui, dans les deux cas, est mise en ouvre. C'est ce qu'il faut maintenant développer.

À la réalité substantielle du divin qui, dans la Phénoménologie, agissait comme sol ou terrain pour la naissance de l'élément tragique, - la conscience de soi comme force de suppression qui doit gagner sa propre suppression, - à cette réalité substantielle du divin analysée comme réalité présente dans les animaux, les forces multiples répandues dans la nature, le soleil, l'opacité de la pierre, etc., correspond, dans l'Esthétique, l'élément épique, c'est-à-dire l'esprit présent dans le monde qui a sa base, sa réalité, sa tenue et sa cohésion dans le peuple. Le peuple forme ainsi le fond réel national, peut-être conviendrait-il mieux de dire : " nationel ", car se réalise en lui l'union de la langue et de la représentation, la langue devenant, de cette union, la matière même de l'élément poétique. Le peuple comme base forme ainsi ce. sur fond de quoi se découpent les grands exploits qui à la fois le façonnent comme unité à la recherche d'elle-même et l'ouvrent au jeu multiple des forces éparses qui, répandues, constituent la totalité du cosmos. Dans le monde épique, nous avons de grandes individualités - Achille, Patrocle, Ulysse, etc. - dont la singularité est d'être, pourrions-nous dire, du jeu des particularités par quoi se manifeste au mieux l'ampleur de la conception cosmologique qui est mise en ouvre dans l'épopée. Il faut ici insister : aux trois moments " logiques " que sont l'universel, le particulier et le singulier, l'épopée se caractérise principalement en ceci que les grandes individualités singulières qui nous sont présentées sont à ou depuis

12. Physique II - 194 a $30-33$.

13. Ce qui correspond à ce que $\mathrm{M}$. Blanchot, dans l'Espace Littéraire, nous apprend à penser : à partir du XIXe siècle, dans ses manifestations les plus dures, l'art se transforme pour devenir passion absolue et quête infinie de lui-même. 
l'universel qu'elles re-présentent par l'intermédiaire d'un jeu de particularités qui à la fois donne à la singularité de ces individualités son caractère pour ainsi dire concret et immédiat, et à l'universel la présence, la réalité et l'ampleur de quelque chose comme un monde. Dans l'épopée, et c'est là son propre, le détail - l'arc de Philoctète, le talon d'Achille, l'olivier d'Ulysse, etc. est manifestation immédiate de la réalité tangible de l'ensemble, et l'unité conceptuelle qui y est jouée serait beaucoup plus de l'ordre d'une logique de la particularité signifiante ou symbolique directement branchée sur la totalité multiple du cosmos, que de l'ordre de l'univocité d'une logique de l'identité ou encore d'une logique de la contradiction. Là, si l'on veut, dans un tel élément, tous les chemins ne mènent pas forcément à Rome, ou plutôt à Ithaque, ou, s'ils finissent bien par y mener, c'est uniquement pour manifester la multiplicité des détours et des distorsions qui y conduisent et la ruse qu'il a fallu déployer pour y arriver. Or, dans la mécanique conceptuelle qui sera mise en œuvre par Hegel pour analyser la Tragédie, ce à quoi nous allons assister, c'est à un oubli complet, ou à une non-considération totale, de ce jeu possible des particularités au nom de ce que nous pourrions appeler une excession des contraires qui trouve à s'opérer par le branchement direct du singulier sur l'universel, lequel se manifeste comme terme du devoirêtre, de l'exigence morale ou du vouloir absolu, c'est-à-dire, en termes essentiellement philosophiques. En d'autres termes, si l'analyse de la Pbénoménologie préparait la venue du christianisme que la Tragédie annonçait, cette fois il faut dire que la Tragédie préfigure le désir de Socrate qui, dans l'exigence de la répétition du "gnôti séauton" à quoi il se vouera, en accomplira vraiment l'“ idée ». Par la Tragédie, nous aurions en quelque sorte une laïcisation de l'esprit païen en vue de sa préparation à l'égard du questionnement philosophique. Cette laïcisation de l'esprit païen n'est pas aussi étrangère qu'on pourrait le croire à la naissance ultérieure du christianisme. En tous les cas l'isomorphie des traitements et des lectures en fonction des termes anticipés permettrait de parler de quelque chose comme un gréco-christianisme dont la structure serait très différente de ce que l'on a coutume d'entendre sous les traits du judéo-christianisme. La Phénoménologie attendait de la religion esthétique une religion révélée dont la raison pouvait prendre la relève, l'Esthétique attend de la Tragédie, comme d'ailleurs de toute forme d'art, le moment, le lieu et l'oc- 
casion de son passage vers le concept ${ }^{14}$. Dans l'un et dans l'autre, dans l'un comme dans l'autre, l'esprit trouve à réaliser sa pâque spéculative. Le plein élément de sa sursomption selon une ligne de fuite infinie dont les termes commencent en effet d'être clairs.

S'il en est ainsi, s'il peut en être ainsi, cela présuppose un statut tout à fait particulier pour les personnages et les situations qui sont mis en scène par la Tragédie, ou encore, dont la Tragédie est la composition globale. Plus précisément, les personnages et les situations seront construits de façon telle que puisse se réaliser au mieux la perspective générale que la grande toile de fond que nous avons développée implique. Dans la Tragédie, les personnages seront donc des êtres qui ne sont qu'à la question de leur propre affirmation, celle-ci étant tout aussi bien celle de l'affirmation de leur être propre et de leur propre constitution dans les termes de ce que nous appelons un propre, puisque ce qu'ils désirent c'est uniquement l'affirmation de la réalité substantielle de la raison et de la justification de leurs actions. Ce sont des êtres qui sont au monde par représentation, ou dont le monde est le terme renversé, et ainsi posé, de l'objectivité de leurs actions. "Ce que nous voyons devant nous ce sont des buts individualisés sous la forme de caractères vivants et de situations riches en conflits, le tout tendant vers un apaisement final. " "La lecture de la Tragédie, c'est-à-dire la définition du sens du tragique par le biais du heurt et de la mise en conflit de buts individualisés, est ainsi rendue possible par l'utilisation de la notion hégélienne de caractère: littéralement, le personnage tragique est l'individualité qui est intégralement transie, qui est complètement possédée par l'unique question de sa propre détermination, celle-ci devant en outre viser sa propre justification. "L'individu tragique est celui qui s'étant proposé un but particulier en fait le contenu pratique de son moi agissant. ${ }^{16} \mathrm{Ce}$ moment de la détermination, c'est-à-dire ce moment de l'inscription intégrale de l'individualité dans les termes de ce que permet d'articuler le rapport fins-moyens-buts, est le plus important, plus précisément il s'agit là de la cheville ouvrière de l'analyse hégélienne par quoi, d'une manière apparemment contradictoire, il sera possible à Hegel de dire d'une part que l'، individu devient le

14. Introduction à l'Esthétique, p. 61-71.

15. Hegel, Esthétique La Poésie, II, p. 322.

16. Hegel, Esthétique, La Poésie II, p. 324. 
centre de la présentation dramatique ${ }^{17}$, et, d'autre part, que l'individu n'est finalement que "le porteur, ou l'organe vivant, de la fin affirmée qui se joue en lui $»^{18}$. Il faut, croyons-nous, insister sur cette étrangeté : comment quelque chose comme un simple porteur, fût-il un organe vivant, peut-il devenir le centre? De deux choses l'une, en effet: ou bien l'individu est effectivement le centre de l'action tragique, et il faut alors voir en lui plus qu'un simple porteur, ou bien il est effectivement le porteur d'un mouvement de finalité qui se joue à travers lui, et c'est alors ce mouvement et son déroulement qui sont le centre de la présentation dramatique. Or, chez Hegel, le fait de mettre ensemble ces deux conceptions très différentes a une fonction précise et qui est d'expliquer l'effet tragique. Cet effet sera le produit ou le résultat de la mise ensemble de ces deux conceptions. Où l'une sera jouée, produite, montrée et représentée, et où l'autre, jouant en dessous, sera la source de la fonction véritablement apaisante de la tragédie, ce qui sera la façon hégélienne de reprendre la catbarsis aristotélicienne. Et regardons-y d'un peu plus près. La mise ensemble de ces deux conceptions - l'individu qui se fixe des buts et devient ainsi le centre; l'individu qui n'est finalement que le porteur d'une finalité qui se joue à travers lui - permettra en effet à Hegel d'articuler un double processus : d'une part, sur la scène ou dans la composition de la Tragédie, il suffira d'avoir un seul personnage qui se détermine par la question de sa propre détermination, un seul personnage qui se fixe un but et s'assigne de le réaliser, qui consente à jouer son être de l'affirmation du but qu'il s'est proposé, pour que tous les autres soient forcés de jouer leur être de la même façon ; naît ainsi la dimension du conflit, c'est-à-dire l'opposition simple et directe des buts et des fins qui dans, par et à travers la tragédie sont amenés à se rencontrer ; à la base de cette conception, il y a donc un pur désir d'ostentation comme confirmation et affirmation de la "réalité " du but poursuivi ; d'autre part, par l'affirmation de ces buts, nous n'avons pas seulement des individus qui visent leur propre affirmation et expression, comme c'est le cas, pour Hegel, de l'individualité lyrique ou de la tragédie romantique, nous avons des individus qui veulent et entendent que le but

\footnotetext{
17. Hegel, ibid. Souvenons-nous ici d'Aristote, sur lequel nous reviendrons et pour qui ce qui peut répondre de notre catégorie de l'individu, la notion d'èthos est et n'est qu'un effet et une fonction de l'action tragique.

18. Hegel, Esthétique, La Poésie, II, p. 326.
} 
dont ils poursuivent l'achèvement soit justifié en soi et pour soi. En plus de n'avoir de sens que pour eux, ce qui ne serait que pure et simple expression de subjectivité, ces buts poursuivis doivent aussi avoir, pour parler comme Descartes, de la "réalité objective ". Et on pourrait même à cet égard donner le principe qui permettrait de fonder une hiérarchisation ou une sélection possible des tragédies : plus le but poursuivi par un individu a de "réalité objective ", plus le but poursuivi a de " substance ", plus la situation peut être tragique. L'opposition de Créon et d'Antigone, par exemple, est de ce type : c'est la réalité ou la substantialité de l'idée de la loi ellemême qui par les deux personnages se trouve radicalement mise en cause. Etc. Pour qu'une telle opposition - la plus dure - au sein du même posé objectivement fonctionne, et pour son fonctionnement, nous avons donc un double mouvement : d'une part, il faut que nous ayons des êtres véritablement conscients, c'est-àdire, des êtres capables de se déterminer et d'être parfaitement responsables de leurs actes, en d'autres termes, des " sujets " au sens cartésien du terme ; d'autre part, il faut que ce pour quoi ils se déterminent soit objectivement valable, c'est-à-dire soit l'expression, au sens plein et cette fois spinoziste du terme, de la réalité substantielle de leurs actions. Dans la Phénoménologie, c'est l'aspect substantiel du divin qui, se jouant ainsi à travers l'action de l'humain, se voit affirmé ; dans l'Estbétique, c'est la morale, le divin qui est le même que la morale formant ce côté substantiel de l'action : "C'est la morale qui constitue la substance spirituelle du vouloir et de sa réalisation (. . . ) car le moral, c'est le divin dans sa réalité profane, le substantiel dont les côtés fournissent un contenu capable de mettre en mouvement l'action véritablement humaine, etc. " $"$ On voit dès lors le processus qui est mis en œuvre dans la tragédie, ou, dont la tragédie est l'expression, un processus qui peut être envisagé d'une double façon : du côté de la substantialité divine ou morale - nous ne les distinguerons plus - la Tragédie représente une scission de la substantialité elle-même ; c'est, pour Hegel, le caractère insupportable, cela donc qui, par suppression simple, doit être ramené au frais de l'identité calme, car « les dieux ne remplissent donc leur véritable mission qu'aussi longtemps que séjournant dans l'Olympe et dans le ciel de l'imagination et des représentations religieuses, ils jouissent d'un calme et d'une unité

19. Hegel, Esthétique, La Poésie II, p. 375. 
paisibles $"^{20}$; et du côté de cet être, le personnage, dont tout l'être, semble-t-il, s'est joué devant nous, la Tragédie représente une destruction de l'individualité qui s'est ainsi élevée jusqu'à s'identifier à la substance morale ellę-même; c'est là, pour Hegel, l'aspect apaisant ou reposant de la Tragédie, c'est-à-dire le moment du retour à l'unité intiale, ce retour qui s'obtient par la suppression de l'unilatéralité. La faute, pourrions-nous dire, ce qui doit être supprimé, c'est, littéralement, ce qui procède de cela même dont tout a procédé : le sujet de ce pouvoir de décision et l'unilatéralité nécessaire qui s'est alors et ainsi manifestée. La formule de cette suppression nous paraît être la suivante : toute action doit avoir un but; toute action qui a un but suscite nécessairement devant elle une autre action qui a un autre but; la justification même de ces actions et de ces buts entrant en conflit les uns avec les autres et menaçant l'idée même de justification, c'est la justification ellemême et ce qu'elle représente - l'idée du droit, de la morale, de la justice, etc. - qui exigent la suppression et la destruction de ce qui fut la cause de la provocation: l'individu qui s'estime en position de sujet.

La substance doit aussi être sujet, disions-nous au tout début, répétant alors ce qui, pour la Pbénoménologie, vaut et est posé ${ }^{21}$ comme le principe même de la méthode. Dans la Tragédie, ce à quoi nous assistons, c'est en quelque sorte à la naissance d'un pur sujet qui soit véritablement capable de substantialité. Mais la Tragédie faute, elle manque, elle est expérience inachevée, car le sujet qui s'y joue, sous les figures, par exemple, d'Oedipe, d'Antigone, de Créon, etc., a encore besoin de l'extériorité formelle de la substantialité abstraite. Ce à quoi nous allons alors assister, c'est à la naissance d'un sujet qui est dans le désir de la substantialité, à la naissance d'un sujet qui voudrait aussi être substance ; la substance elle-même est alors menacée dans sa substantialité et exige la destruction de ce qui avait tenté de s'élever jusqu'à elle. L'aspect horrible de la Tragédie est que le divin lui-même paraît vaciller sur son socle, que la substance elle-même paraît être scindée. Le mouvement de destruction par quoi l'unité perdue se regagne peut être envisagé en un double sens : du côté de l'humain, la suppression des vies et le sacrifice des individualités sont affirmation de la

20. Ibid., p. 376 .

21. Hegel, Phénoménologie 1, p. 17. 
réalité de la substance, du principe même de la justification et de l'idée du droit ; c'est même très précisément cette réalité qui se manifeste au mieux par la suppression de ce qui la menace ; du côté de la substance divine, cette scission dans le sein de la substantialité qui exige sa réparation et son retour à l'unité n'est qu'une manifestation de la courte vue de l'humain, courte vue qui peut et doit, sans perte et sans risque, être supprimée. Mais dans ce jeu, pour le divin lui-même, c'est-à-dire avant que, pour Hegel, ce divin ne monte lui-même sur la croix pour accomplir la subjectivité fondamentale de la substantialité divine, on va du repos au repos, du silence au silence, de la quiétude à la quiétude, de la sérénité paisible à l'inactivité heureuse. "Ces puissances ne réalisent l'œuvre de l'esprit au sein d'une vie sereine qu'aussi longtemps qu'elles restent à l'état inactif qui est celui des dieux bienheureux. "12 Par la Tragédie, une pierre a été lancée dans cette mare calme de la sérénité, le repos des dieux a été troublé, des rides sont apparues à la surface d'une eau qui exige le retour unifié de sa limpidité.

On pourrait se servir de ces passages pour entamer une critique de Hegel et remarquer qu'il ne s'est agi, dans cette étude de la tragédie, que du parcours et du tracé d'un cercle de plus dans l'ensemble de ces anneaux dont la totalité constitue ce que Hegel appelle l'Esprit ${ }^{23}$. On pourrait redoubler la métaphore et remarquer que s'est à nouveau exercé le plaisir de lancer des pierres à l'eau pour y retrouver les cercles concentriques dont "l'esprit comme l'enfant est aussi l'auteur ${ }^{24}$. Ce serait une autre façon de souligner qu'à nouveau l'esprit s'est épousé lui-même et qu'il ne retrouve partout ce que qu'il y met : la forme de l'anneau qui le rappelle à lui-même et à ce reflet idéal d'un soi pur qui, depuis Platon $^{25}$, ayant retourné toutes les surfaces, ne saurait plus être

22. Hegel, Esthétique, La Poésie II, p. 376.

23. "La philosophie de l'art forme un anneau nécessaire dans l'ensemble de la philosophie... Dans cette couronne formée par cette nécessité scientifique, chaque partie représente un cercle revenant sur Jui-même..." Introduction, p. 17.

24. Puisque ce besoin, ce narcissisme originaire, est posé comme fondement du " besoin d'art ": "On saisit déjà cette tendance dans les premières impulsions de l'enfant : il veut voir des choses dont il soit lui-même l'auteur, et s'il lance des pierres dans l'eau, c'est pour voir des cercles qui se forment et qui sont son ouvre dans laquelle il retrouve comme un reflet de luimême... "Introduction à l'Esthétique, ibid., p. 81.

25. Cf. entre autres les passages du Phèdre sur l'ophtalmis produite par le miroir, $255 \mathrm{~d}$; auxquels, pour strictes fins de contraste, on peut opposer les passages célèbres de République $\mathrm{X}$ sur la fonction du miroir. 
troublé. Nous aimerions plutôt proposer autre chose : indiquer en direction de ce que nous appelons un "foyer de sens ", et, ce qui plus est, indiquer ce foyer principal de ce qui est censé faire sens, qui commande toute l'analyse hégélienne et qui est partout inquestionné. Sous la catégorie de l'action que Hegel met en œuvre pour interpréter la Tragédie, soit sous la catégorie de l'action tragique, il y a en effet ce postulat suivant lequel toute action procède d'un être qui en est le sujet, la cause, l'auteur, le principe, l'élément responsable, etc. L'action ainsi comprise est le moyen pour ce sujet de se réaliser, c'est-à-dire d'accomplir le but, le motif, la fin ou l'intention qu'il s'était proposé, ou, à l'obtention de quoi il s'était résigné ou décidé. En d'autres termes, l'action ainsi comprise est le lieu, comme moment de passage, où s'accomplit l'adéquation du caractère, ce qui est tout aussi bien sa réalisation complète comme désir du but qu'il s'était proposé, que sa soumission tout aussi complète à l'ordre ou au règne des fins qui ainsi, et peut-être ainsi seulement, trouve à advenir : le propre d'une situation véritablement tragique, comme nous l'avons vu, est que dès qu'un être entreprend d'entrer dans ce jeu, - se fixer un but par l'obtention ou la réalisation duquel il devient, - il force tous les autres à faire de même; l'objectivité des fins ou des buts poursuivis se trouve alors mise en cause, etc. L'action, en ce sens, que ce soit du point de vue du personnage qui à travers elle joue la vérité de son être, que ce soit du point de vue de l'auteur ou du poète qui la compose, ou que ce soit enfin du point de vue du spectateur ou du critique à qui elle est donnée à voir, - les trois situations sont en effet idéalement censées être isomorphes, - l'action devient ainsi en quelque sorte l'équivalent de la mineure d'un syllogisme grâce à laquelle je puis passer de la majeure à la conclusion. La Tragédie la mieux réussie est alors celle en et par laquelle ce " passage " est au mieux accompli, et ce passage est au mieux accompli lorsqu'il est au plus près, au point d'ailleurs de pouvoir s'y confondre ou d'en être la doublure, de la définition traditionnelle de la vérité entendue comme "adaquatio". D'une pièce représentée, je puis alors parler de la "vérité " de ses caractères, de la "réalité substantielle " des buts qui y sont poursuivis, de la "correspondance" des situations aux intentions, du degré d'" adéquation" de la forme au contenu, etc. Or l'action ainsi comprise, comme provenant essentiellement d'un être qui est posé dans l'être comme un être et dont elle est l'" expression ", que celle-ci le soit de son caractère ou de la réalité subs- 
tantielle du but qu'il poursuit, etc., ${ }^{26}$ l'action ainsi comprise est la conception moderne par excellence, dont Hegel représente ici le terme ultime, qui n'est pourtant point final, et dont il faut entreprendre la déconstruction. La raison en est simple : s'il ne nous est pas loisible, faute d'espace dans un article où de surcroît ce n'en serait pas le lieu, d'entreprendre de démontrer comment et pourquoi une telle conception est peut-être ce qu'il a de plus incompatible au développement d'une véritable conception de l'action tragique, autre du moins que celles qui procèdent de la répétition des schémas du psychologisme ${ }^{27}$, il est toutefois possible de montrer que cette conception procède d'un véritable, renversement de la conception aristotélicienne d'où, d'une part, elle provient - ce qu'elle reconnaît - et où, d'autre part, elle est pourtant radicalement exclue. La situation peut paraître paradoxale, elle n'est pourtant pas unique ${ }^{28}$; il faut cependant l'établir dans le but de montrer comment, étant guidée par un foyer principal de ce qui est censé faire sens, l'analyse de Hegel n'en effectue pas moins un détournement et assure ainsi une imcompréhension fondamentale de ce qui était proposé par Aristote. Ainsi serons-nous amenés à mettre à jour la limitation principale de ce foyer de sens, à tenter d'en signer la clôture, et à en réouvrir la question.

En Poétique ${ }^{29}$, après avoir distingué les six parties constitutives de ce que nous appelons une Tragédie, soit le "mutbos", les personnages ou caractères (èthos), la pensée, c'est-à-dire le langage approprié à la situation, l'élocution (lexis), c'est-à-dire l'art de rendre les vers, le chant et le spectacle, Aristote nous donne le texte suivant qui à notre avis est le plus important car s'y

26. On pourrait poursuivre puisque ce même schéma est ce qui permet et fonde l'insertion de la psycho-critique et de la psychanalyse dans le champ de l'ceuvre ; l'action devient alors manifestation exemplaire de l'inconscient du personnage, ou, par transport, de son auteur ; elle peut également devenir expression de sa situation particulière par rapport à l' "idéologie "dominante, etc.

27. Qu'une telle conception soit sinon incompatible du moins représente un obstacle - au sens bachelardien - au véritable développement d'une conception de l'action tragique, on peut l'indiquer rapidement à ceci que les trois critiques les plus radicales et les plus importantes de l'impossibilité du théâtre actuel dénoncent ce schéma conceptuel où littéralement l'action n'y est pour rien. C'est là, en effet, ce que Brecht dénonce dans sa critique de l'identification ; c'est également là ce que Nietzsche et Artaud critiquent sous le nom de " psychologisme".

28. P. Aubenque avait en effet démontré qu'on n'avait pas le droit d'étendre à l'être ce qu'Aristote ne mentionnait qu'à propos du Bien. Cf. Le Problème de l'Être chez Aristote, Paris, P.U.F., 1966, p. 202 à 206.

29. Poétique 1450 a, 15-26. Traduction Budé, légèrement modifiée. 
condense la théorie aristotélicienne de l'action tragique. On nous permettra donc de le donner tout au long en le commentant:

« La plus importante de ces parties est la composition des actions accomplies (è tôn pragmatôn sustasis); car la tragédie est l'évocation (mimésis) ${ }^{30}$ non pas d'hommes, mais de l'action (praxéôs) ${ }^{31}$ et de la vie (Biou), et du bonheur et du malheur; or le bonheur et le malheur sont dans l'action, et non pas dans une manière d'être. C'est en raison de leur caractère (kata ta èthè) que les hommes sont tels ou tels - (c'est-à-dire qu'ils sont identifiables ou reconnaissables, qu'ils ont leur " différence spécifique ", en d'autres termes : qu'ils peuvent relever de la catégorie qui permet l'identification) - c'est en raison de leurs actions (kata tas praxeis) qu'ils sont heureux ou le contraire. Donc les personnages n'agissent pas pour évoquer ou rendre (mimèsontai) leur caractère, ils reçoivent bien plutôt leurs caractères de surcroît, et comme par effet de retour de l'action sur elle-même (sum-péri-lambanousi), à travers leurs actions (dias tas praxeis). Ainsi, les actions et le muthos sontils la fin (télos) de la Tragédie et en toutes choses la fin est-elle le principal. De plus, sans action il ne peut y avoir de Tragédie, mais il peut y en avoir sans caractère (a-ètheis). En effet, les Tragédies de la plupart des auteurs récents sont dépourvues de caractères, etc. " $^{32}$

Le texte est donc clair : l'action est un registre de passage, un mouvement d'inscription et d'accomplissement de tels registres de passages qui, dans leur effectuation ou leur effectivité, ce que les Allemands appellent le "wirklicbkeit", sont radicalement indépendants et théoriquement disjoints d'une théorie du sujet, ou de l'homme comme d'un être posé dans l'être qui en serait la cause, l'auteur, l'élément responsable, etc. L'analyse d'Aristote n'a ainsi pas besoin d'un concept du " héros tragique " comme terme posé, figure exemplaire, père et auteur des actions qu'il accomplit et qui le révèlent dans sa nature ou ses intentions, concept du " héros tragique " dont, quoiqu'on en ait dit, la notion n'apparaît même pas dans la Poétique ${ }^{33}$. Ce qu'Aristote nous donne par contre à penser,

30. Nous laissons aux philologues l'entière et unique responsabilité de la traduction de la notion de mimèsis par celle d'imitation.

31. Nous n'avons ici qu'un seul terme, celui d'" action ", pour distinguer les deux notions de "praxis" et de "pragmata".

32. Pour les problèmes philologiques posés par ce passage, cf. principalement Jones, On Aristotle and the Greek Tragedy, Chatto and Vindus, Londres 1967.

33. La remarque, croyons-nous, est de taille et vaut d'être soulignée. Malgré le sous-titre qu'après tant d'autres J. Hardy donne au second paragraphe du chapitre 13 - "Ce que doit être le héros" - (cf. Poétique, Éd. G. Budé, p. 47), la notion de héros, en grec "héròs", est absente de la Poétique. Elle y est mise par les traducteurs parce qu'il s'agit là pour nous d'un 
c'est ce que nous appellerions l'irréductibilité d'une logique de l'action tragique à ce que peut permettre d'en penser (d'en déduire) une logique du sujet comme terme posé dans l'être d'un être qui, à travers un certain nombre de processus psychologiques, d'intentions, de volitions, d'intérêts, de buts, voire de désirs, se mettrait à agir. L'ordre de l'action serait ainsi incommensurable à l'égard de ce qu'il pourrait en être d'une logique de l'être qui d'ailleurs pourrait très bien n'être, soit qu'une hiérarchie de type platonicien, dont nous avons retrouvé et indiqué certaines traces chez Hegel, soit que la présomption de sa soumission complète à un " ordre des raisons" de type cartésien. Car Aristote non seulement nous a montré qu'une logique de l'action n'avait pas besoin, pour être développée, d'un quelconque fondement posé dans l'être, mais encore a-t-il insisté pour dire, et répéter, que cette logique est à elle seule responsable de ce qu'il pourra en être de cet être et de ce qu'on pourra en dire. Ce que nous appelons le sujet d'une action, en étant porté à penser ce sujet comme la cause ou le principe de l'action qu'il produit, Aristote nous donne à penser tout au contraire qu'il en est l'effet strict. Ce qu'il peut en être de ce sujet, de son être, de sa nature et de ce qu'on peut en dire, ce qui peut nous le faire reconnaître et dire qu'il est tel ou tel, donc ce à partir d'où le jeu de l'" identification" devient possible, cela procède uniquement de l'action qui en produit les conditions. Répétons en effet ce passage : "La Tragédie est une évocation (mimèsis) non pas des hommes, mais de l'action." - "Les hommes reçoivent leurs caractères de surcroît et comme par effet de retour (sum-péri. lambanousi) de l'action, ou à travers leurs actions. i" ${ }^{34}$ La Tragédie, pour Aristote, est une composition globale d'actions de ce genre, c'est-à-dire d'actions qui, "littéralement et à tous les sens du terme ", pour reprendre un mot de Rimbaud, fasonnent des êtres, mais, fondamentalement, ce n'est pas là, pour lui, le plus important. En d'autres termes, à trop insister sur cet aspect, nous donnerions à son texte une pente métaphorique existentielle où le penchant existentiel ne serait peut-être que l'une des versions du sym-

foyer principal de ce qui est censé faire sens. Sur la question, et sur celle de son impact sur le terrain de la philologie, cf. les remarques de Jones, t.c. p. 12 sq. Cf. également le texte de Vernant : "Ébauches de la Volonté en Grèce Ancienne ", in Mythe et Tragédie, Paris, Maspéro, 1973.

34. Et sur la question de la prédominance de l'agir dans la détermination de l'être, qui est une thèse classique chez Aristote, on peut aussi consulter les passages suivants d'Aristote: Physique 197 b 4 ; Politique 1325 a 32 ; Étbique à Nicomaque 1098 a 16, b 21, etc. 
bolisme, nous l'animerions de la volonté d'un " télos " qu'il exclut. L'action tragique, en effet, n'a pas pour fonction, terme, fin ou but de nous montrer les êtres qu'elle façonne, de les rendre ostensibles ou de les installer en position de figures exemplaires pour nous faire jouir de l'effet produit ; il faudrait dire de l'action tragique comme pure inscription de passages et de mouvements que tantôt elle peut avoir un tel effet et que tantôt elle ne l'aura pas, l'essentiel de toute façon n'étant pas de ce côté, mais bien plutôt et uniquement du côté du renversement (métabolè) dans le sens ou la direction des actions accomplies, ces revirements dont les formes principales sont appelées péripéties et reconnaissances. La force d'entraînement (psychagogei) dont joue la Tragédie, écrira en effet Aristote (1450 a 33-35), se trouve principalement dans " les parties du muthos, je veux dire dans les péripéties et les reconnaissances "; et c'est par ces péripéties et ces reconnaissances qui, répétons-le, sont uniquement des renversements ou des revirements dans le sens ou la direction des actions ${ }^{35}$, que sont produits Pitié et Crainte, Éléos et Phobos, qui en sont également l'effet strict ${ }^{36}$. Terminons ce commentaire par une remarque : si le christianisme nous a habitués à entendre la pitié comme portant sur des êtres, ou si, pour être effective, sinon efficace, la pitié doit porter sur des êtres ${ }^{37}$, il faut alors dire qu'elle est un détournement de l'Éléos aristotélicienne qui ne portait que sur des relations; ou, peut-être peut-on également dire qu'elle en est à la fois l'application et l'accomplissement, dans la mesure où Aristote nous a montré que ce à partir d'où ces êtres pouvaient recevoir la qualification de leur être, dans la Tragédie à tout le moins où la possibilité de cette qualification est jouée, était un effet strict de cet ensemble de relations dont, comme association d'actions, la Tragédie est la composition.

Or, de ce texte, Racine nous donne la traduction et le commentaire suivants :

"La Tragédie est l'imitation d'une action. Or toute action suppose des gens qui agissent, et les gens qui agissent ont né-

35. Poétique 1452 a, 22-31.

36. Ibid, 1452 b 28 à 1453 a 7 .

37. Tel est en effet le principe qui anime et détermine la condamnation augustinienne du théâtre : comment puis-je accepter de voir réalisé fictivement sur la scène ce que par là même je m'interdis de réaliser et d'accomplir dans ma vie? Qu'est-ce là sinon une "étonnante folie " ? Etc. Cf. Confessions, livre III. Nous avons commenté ailleurs ces passages. Cf. "Aristote et la Poétique ", in Études littéraires, vol. 9, no 3, décembre 1976, p. 555-578. 
cessairement un caractère, c'est-à-dire : des mœurs et des inclinations qui les font agir ; car ce sont les mœurs et l'inclination, c'està-dire: la disposition de l'esprit, qui rendent les actions telles ou telles ; et par conséquent les mœurs et le sentiment, ou la disposition de l'esprit, sont les deux principes des actions. Ajoutez que c'est par ces deux choses que tous les hommes viennent ou ne viennent pas à bout de leurs desseins et de ce qu'ils souhaitent ${ }^{38}$

Ainsi, pour Racine, qui prend ici le contre-pied d'Aristote, loin d'être ontologiquement première et décisive dans la détermination de ce que nous appelons un être, l'action n'a-t-elle pour fonction que d'être le milieu, et un milieu, qui, à la limite, ne peut être que transparent, car, à la lettre, rien ne s'y produit à travers lequel se réalise l'adéquation du " caractère ". En termes hégéliens, elle est le lieu matriciel, et, comme toute matrice, essentiellement disparaissant, où se conquiert, se pose, se gagne et s'affirme ce que l'" Esprit ", c'est-à-dire le désir de rationalité, savait et attendait depuis le début : le devenir soi d'une figure du soi. Nous désirions au tout début mettre à jour les éléments principaux de la machine de lecture que Hegel met en œuvre à propos de la Tragédie, et, plus précisément, en prenant l'étude de la Tragédie comme exemple particulier, interroger l'étrangeté même de ce type de discours qui interroge l'art à partir du point anticipé de sa disparition ou de sa relève. Peut-être est-il alors possible de demander si l'art, plus particulièrement cette forme de l'art qui, comme la Tragédie ou la poésie, parce qu'elle est de la parole, peut être dans l'élément de la représentation, n'est pas essentiellement destiné à disparaittre, ne doit pas nécessairement disparaitre, lorsqu'il est lu, perçu ou considéré par ce désir de rationalité qui n'en attend que sa propre constitution, ou la répétition seule de ce qui l'assure constamment de lui-même. Quoiqu'il en soit, ce qu'il importe ici de remarquer, c'est que l'analyse hégélienne s'inscrit dans le cadre de ce qu'illustre particulièrement l'interprétation racinienne, interprétation qui, en étant présentée comme une " traduction" du texte d'Aristote, est bien plutôt d'inspiration strictement cartésienne. Pour ce type de pensée, en effet, une action ne peut être comprise qu'à partir et sur le fondement de quelque chose comme un être qui est posé dans l'être comme un être et de ce qui est ainsi, mais de façon tout à fait extérieure à cet être, conceptuellement et logiquement

38. Racine, auvres Complètes, L'Intégrale, éd. du Seuil, 1962, p. 587. En commentant ce passage, Geoffroy écrivait : "Le style de ce philosophe (i.e. Aristote) étant très concis, Racine s'est permis quelques paraphrases en faveur de la clarté " ? 
rendu possible. Il resterait bien sûr à nous demander si le jeu des schémas logiques, qui est ainsi ouvert et offert au calcul, est effectivement conforme à, ou peut effectivement répondre de, et alors à quel prix, cette liasse de relations dont la seule forme grecque de la Tragédie peut toujours être la question et l'occasion. Il nous suffisait de mettre en lumière ce postulat qui, tel un foyer de sens, commande et régit l'analyse hégélienne ; mais, et dans l'unique but de tenter d'ouvrir quelque peu l'espace qui pourrait rendre à nouveau possible cette question que peut nous être la Tragédie, nous aimerions terminer par la citation d'un dernier passage d'Aristote où se trouve exclue, comme ne répondant pas de ce qui est l'« œuvre propre " de la Tragédie, l'interprétation racinienne. Il se peut que le penchant au psychologisme réponde, pour reprendre les termes de Foucault, d'une configuration finalement récente de ce qui en nous s'appelle savoir ; Aristote nous offre peut-être encore autre chose, un élément au moins de comparaison et l'indication d'une filiation : "En outre, si on met à la file des tirades qui reflètent un caractère, si bien réussies qu'elles soient sous le rapport de l'élocution et de la pensée, on n'effectuera pas ce qui est l'œuvre propre de la tragédie (en tès tragodias ergon), mais on l'effectuera bien davantage avec une Tragédie inférieure sous tous ces rapports, mais ayant "muthos" et composition d'actions. " Si la Tragédie pouvait toujours nous être question, ou si, ce qui revient au même, dans quelque chose comme une scène pouvaient toujours être jouées des figures et des structurations de ce qui nous est possible, et ce qui plus est, s'il était possible d'en produire devant nous l'impossibilité, le primat de l'action seule, avant toute prédétermination de ce qu'il doit en être de l'être, ne devrait-il pas alors être pris en considération? Nous pourrions ainsi tenter d'en finir avec l'être, comme le demandait Artaud, mais il faudrait également que nous gardions fidèlement à la mémoire, sinon tout près du corps, que les deux théories philosophiques les plus accomplies que l'Occident a produites sur la Tragédie, ou sur son dos, l'ont tenté. Peutêtre alors conviendrait-il de demander s'il ne faut pas choisir entre le désir de l'Aufbebung et l'exigence d'un pessimisme encore plus radical que la radicalité même de tout ce qui a été fait à partir de ce que l'on pouvait souhaiter? Oedipe, du moins, savait des choses làdessus.

Université de Montréal

39. Poétique, 1450 a, 29.35. 